\title{
YAP signaling in gastric cancer-derived mesenchymal stem cells is critical for its promoting role in cancer progression
}

\author{
ZHAOJI PAN $^{1 *}$, YIQING TIAN $^{1 *}$, BIN ZHANG $^{1 *}$, XU ZHANG $^{1}$, HUI SHI $^{1}$, ZHAOFENG LIANG $^{1}$, PEIPEI WU $^{1}$, \\ RONG LI ${ }^{1}$, BENSHUAI YOU ${ }^{1}$, LUNYU YANG ${ }^{1}$, FEI MAO ${ }^{1}$, HUI QIAN ${ }^{1}$ and WENRONG XU ${ }^{1,2}$ \\ ${ }^{1}$ Jiangsu Key Laboratory of Medical Science and Laboratory Medicine, School of Medicine, Jiangsu University, \\ Zhenjiang, Jiangsu 212013; ${ }^{2}$ The Affiliated Hospital, Jiangsu University, Zhenjiang, Jiangsu 212000, P.R. China
}

Received May 11, 2017; Accepted July 24, 2017

DOI: $10.3892 /$ ijo.2017.4101

\begin{abstract}
Cancer-associated mesenchymal stem cells (MSCs) are critically involved in tumor development and progression. However, the mechanisms of action for MSCs in cancer remain largely unknown. Herein, we reported that the expression of Yes-associated protein 1 (YAP) was higher in gastric cancer derived mesenchymal stem cells (GC-MSCs) than that in bone marrow derived MSCs (BM-MSCs). YAP knockdown not only inhibited the growth, migration and invasion, and stemness of GC-MSCs, but also suppressed their promoting effect on gastric cancer growth in vitro and in vivo. In addition, the interference of YAP expression in GC-MSCs also attenuated the promoting role of gastric cancer cells in endothelial cell tube formation and migration. Mechanistically, YAP knockdown reduced the activation of $\beta$-catenin and its target genes in gastric cancer cells by GC-MSCs. Taken together, these findings suggest that YAP activation in GC-MSCs plays an important role in promoting gastric cancer progression, which may represent a potential target for gastric cancer therapy.
\end{abstract}

\section{Introduction}

Gastric cancer is the fourth most common cancer and second leading cause of cancer-related deaths worldwide (1-4). Although great efforts have been made, the current treatments for gastric cancer are still not efficacious (5). Increasing evidence has shown that the microenvironment plays important roles in gastric cancer progression (6,7). Mesenchymal stem cells (MSCs) are a critical component of cancer microenvironment. We have

Correspondence to: Professor Wenrong Xu or Professor Hui Qian, Jiangsu Key Laboratory of Medical Science and Laboratory Medicine, School of Medicine, Jiangsu University, 301 Xuefu Road, Zhenjiang, Jiangsu 212013, P.R. China

E-mail: icls@ujs.edu.cn

E-mail: 1stmmmlst@163.com

*Contributed equally

Key words: Yes-associated protein 1, mesenchymal stem cell, gastric cancer, $\beta$-catenin, progression previously reported the isolation of MSCs from gastric cancer tissues (GC-MSCs) and have demonstrated that these cells could promote gastric cancer growth and metastasis (8-10). However, the underlying mechanisms responsible for the promoting role of GC-MSCs in gastric cancer progression remain unknown.

The Hippo pathway plays a critical role in organ size control, tissue homeostasis and early embryonic cell specification (11). Yes-associated protein 1 (YAP) is the central component of this pathway. YAP has been recently identified as an oncoprotein that is overexpressed in many cancers, including gastric cancer, liver cancer, lung cancer, breast cancer, and ovarian cancer among others (12-14). The knockdown of YAP inhibits gastric cancer cell proliferation, migration, invasion, and metastasis $(15,16)$, suggesting that YAP plays important roles in the development and progression of gastric cancer.

In addition to tumor cells, YAP could also regulate the microenvironmental cells. For instance, YAP could modulate cell-cell and cell-matrix interactions (17) as well as the production of secretory proteins such as amphiregulin [AREG; an epidermal growth factor (EGF) family member], cysteine-rich angiogenic inducer 61 (CYR61), and connective tissue growth factor (CTGF) (18-20). Moreover, YAP expression in the microenvironmental cells also affect tumor progression. YAP is required for the tumor-promoting role of CAFs in matrix remodeling and angiogenesis $(17,21)$, indicating that YAP pathway may affect tumor progression by regulating both tumor cells and microenvironmental cells.

Mesenchymal stem cells (MSCs) have been suggested as a key player in the tumor microenvironment. We have previously shown that MSCs isolated from gastric cancer tissues have a strong promoting role in gastric cancer progression (8-10). However, the detailed molecular mechanism is not clear. In this study, we aimed to investigate whether YAP is involved in the promoting effect of GC-MSCs on gastric cancer progression. We found that YAP silencing significantly suppressed the promoting effects of GC-MSC on gastric cancer growth in vitro and in vivo, which may be associated with the decreased activation of $\beta$-catenin in gastric cancer cells.

\section{Materials and methods}

Subjects. The gastric cancer tissues were collected from 15 patients with gastric adenocarcinoma between August 
2015 and February 2016 in the Affiliated Hospital of Jiangsu University. There were 8 male and 7 female patients with ages ranging from 48 to 71 years old (median, 59 years old). The patients were diagnosed by pathological analyses according to the UICC (International Union Against Cancer) criteria. The use of clinical sample was approved by the Ethics Committee of Jiangsu University and written informed consent was obtained from all the patients.

Isolation and culture of MSCs from gastric cancer tissues. GC-MSCs were isolated as previously described $(22,23)$. Fresh gastric cancer tissues were washed with phosphate-buffered saline (PBS) to remove the blood. Then, the tissues were cut into 1-3 $\mathrm{mm}^{3}$-sized pieces and floated in Dulbecco's modified Eagle's medium with low glucose (LG-DMEM, Invitrogen, Carlsbad, CA, USA) containing 15\% fetal bovine serum (FBS, Invitrogen), penicillin $(100 \mathrm{U} / \mathrm{ml})$ and streptomycin $(100 \mu \mathrm{g} / \mathrm{ml})$. The pieces of gastric cancer tissues were cultured at $37^{\circ} \mathrm{C}$ in humidified air with $5 \% \mathrm{CO}_{2}$. After culturing for 10 days, the colonies of fibroblast-like cells appeared. When the cells reached $\sim 80 \%$ confluence, they were trypsinized and re-plated into larger culture flasks at a 1:3 split ratio. The GC-MSCs at passage 4 were used for subsequent experiments.

Cell culture. GC-MSCs were cultured in LG-DMEM with 15\% FBS. The human GC cell lines SGC-7901 (moderately differentiated), HGC-27 (undifferentiated) and MGC-803 (poorly differentiated) were purchased from the Cell Bank of the China Academy of Sciences (Beijing, China) and maintained in the RPMI-1640 medium (Gibco, Grand Island, NY, USA) supplemented with $10 \%$ FBS. Human umbilical vein endothelial cell line EA.hy926 was purchased from the Cell Bank of the China Academy of Sciences and maintained in high-glucose DMEM supplemented with 10\% FBS. All the cells were cultured at $37^{\circ} \mathrm{C}$ in humidified atmosphere with $5 \% \mathrm{CO}_{2}$.

Lentiviral knockdown of YAP. The lentiviral expression vector containing the shRNA sequence (Sigma) was selected for target-specific gene silencing. The shRNA sequences targeting Yap were as follows: forward, CCGGGCCACCAAGCTAGA TAAAGAACTCGAGTTCTTTATCTAGCTTGGTGGCTTT TTG; reverse, AATTCAAAAAGCCACCAAGCTAGATAA AGAACTCGAGTTCTTTATCTAGCTTGGTGGC. Control shRNAs were constructed using scrambled sequences. The shRNA lentiviral vectors were generated by ligating the vector Tet-pLKO-puro; these lentiviral vectors were produced using a lentivirus packaging mix (ViraPower, Invitrogen). In addition, stable cell line was obtained after selection with $1 \mu \mathrm{g} / \mathrm{ml}$ of puromycin (Invitrogen) for 5 days. The efficiency of YAP knockdown was evaluated by using real-time quantitative RT-PCR and western blotting.

Generation of conditioned medium. Control and YAP knockdown GC-MSCs were plated in 6-well plates at a density of $1 \times 10^{5}$ cells/well and cultured in $1.6 \mathrm{ml}$ complete LG-DMEM with $15 \%$ FBS. After $72 \mathrm{~h}$, the conditioned medium (CM) was collected, centrifuged to remove cellular debris $(800 \mathrm{~g}$ for $5 \mathrm{~min}$ ) and passed through a $0.22-\mu \mathrm{m}$ filter (Millipore, Billerica, MA, USA) and stored in $-20^{\circ} \mathrm{C}$ until use. Gastric cancer cell-derived $\mathrm{CM}$ was generated in a similar manner.
RNA extraction and real-time RT-PCR. Total RNA was isolated from cells and tissues using TRIzol reagent (Invitrogen) according to the manufacturer's instructions, and equal amount of RNA was used for RT-PCR. The StepOne Real-Time system was used for quantitative mRNA detection. The expression of target genes was normalized to that of $\beta$-actin. The expression of each gene was measured by formula $2^{-\Delta \Delta C t}$. The primers used in this study were produced by Invitrogen (Shanghai, China) and the sequences are listed in Table I.

Western blotting. GC-MSCs and gastric cancer cells were homogenized and lysed in RIPA buffer supplemented with proteinase inhibitors. Equal amounts of proteins were separated on a $12 \%$ SDS-PAGE gel. Following electrophoresis, the proteins were transferred to the PVDF membrane, blocked in $5 \%(\mathrm{w} / \mathrm{v})$ non-fat milk and incubated with the primary antibodies at $4^{\circ} \mathrm{C}$ overnight. The sources of antibodies were as follows: anti-GAPDH (Kangcheng, China); anti-YAP, anti-vimentin, anti-Bcl2, and anti-Bax (Bioworld Technology, Louis Park, MN, USA); anti- $\beta$-catenin (Cell Signaling Technology, Beverly, MA, USA); anti-E-cadherin, and antiN-cadherin (Santa Cruz Biotechnology, Inc., Santa Cruz, CA, USA); anti-Ki67 (BOSTER, China). The membrane was washed with Tris-buffered saline/Tween 3 times and incubated with the secondary antibodies (Bioworld Technology) at $37^{\circ} \mathrm{C}$ for $1 \mathrm{~h}$. The signals were visualized by using a Lumina crescendo western horseradish peroxidase substrate (Millipore). The dilution factor for the primary and secondary antibodies was 1:1,000, respectively.

Colony formation assay. GC-MSCs and gastric cancer cells were seeded into 6 -well plates $\left(2 \times 10^{3}\right.$ cells/well) and incubated at $37^{\circ} \mathrm{C}$ in a $5 \% \mathrm{CO}_{2}$-humidified incubator for 8 days. SGC-7901 cells were treated with $2 \mathrm{ml}$ complete medium and GC-MSCs derived CM $(1: 1, \mathrm{v} / \mathrm{v})$. The medium was changed at 3-day intervals. At the end of the incubation period, the cultures were fixed with $4 \%$ paraformaldehyde and stained with crystal violet. The results are the mean values of three independent experiments.

Cell migration and invasion assays. GC-MSCs $\left(5 \times 10^{4}\right.$ cells/well), gastric cancer cells treated with GC-MSCs derived CM $\left(1 \times 10^{5}\right.$ cells/well $)$, and EA.hy 926 treated with $\mathrm{CM}$ of gastric cancer cells $\left(5 \times 10^{4}\right.$ cells/well $)$ were plated into the top chamber of Transwell $(8.0-\mu \mathrm{m}$ pore sized) with serum-free L-DMEM, and medium containing $10 \%$ FBS was placed into the bottom chamber. After incubation at $37^{\circ} \mathrm{C}$ in a $5 \% \mathrm{CO}_{2}$-humidified incubator for $12 \mathrm{~h}$, the cells that remained at the upper surface of the membrane were removed with a cotton swab. The filters were fixed in $4 \%$ paraformaldehyde for $30 \mathrm{~min}$, stained with crystal violet for $15 \mathrm{~min}$, and photographed. The cells were observed under a microscope, and at least five fields of cells were assayed for each group. Each assay was repeated in triplicate. For cell invasion assay, the Transwell chambers were coated with $200 \mu 1$ of Matrigel at a dilution of 1:5 in serum-free medium and the incubation time was extended to $24 \mathrm{~h}$. The remaining procedure was the same as that of cell migration assay. The number of migrated and invaded cells was counted under a microscope (Ti-S, Nikon) and five fields were randomly selected. 
Table I. The sequences of the primers.

\begin{tabular}{|c|c|}
\hline Genes & The sequences of the primers \\
\hline \multirow[t]{2}{*}{$\beta$-actin } & Forward 5'-GACCTGTACGCCAACACAGT-3' \\
\hline & Reverse 5'-CTCAGGAGGAGCAATGATCT-3' \\
\hline \multirow[t]{2}{*}{ E-cadherin } & Forward 5'-CGCATTGCCACATACACTCT-3' \\
\hline & Reverse 5'-TTGGCTGAGGATGGTGTAAG-3' \\
\hline \multirow[t]{2}{*}{$\mathrm{N}$-cadherin } & Forward 5'-AGTCAACTGCAACGTCT-3' \\
\hline & Reverse 5'-AGCGTTCCTGTTCCACTCAT-3' \\
\hline \multirow[t]{2}{*}{ Bax } & Forward 5'-CACCAGCAGATCAT-3' \\
\hline & Reverse 5'-GATCAGTTCCGGCACCTTG-3' \\
\hline \multirow[t]{2}{*}{ BCL2 } & Forward 5'-CTGGGAGAACAGGGTACGATAA- \\
\hline & Reverse 5'-CCCACCGAACTCAAAGAAGG-3' \\
\hline \multirow[t]{2}{*}{ MMP2 } & Forward 5'-CTCGAATCCATGATGGAGAG-3' \\
\hline & Reverse 5'-TACTTCACACGGACCACTTG-3' \\
\hline \multirow[t]{2}{*}{ MMP9 } & Forward 5'-ACGTCTTCCAGTACCGAGAG-3' \\
\hline & Reverse 5'-GGCACTGCAGGATGTCATAG-3' \\
\hline \multirow[t]{2}{*}{ Slug } & Forward 5'-CCTGGTTGCTTCAAGGACAC-3' \\
\hline & Reverse 5'-TCCATGCTCTTGCAGCTCTC-3' \\
\hline \multirow[t]{2}{*}{ Oct4 } & Forward 5'-TTGAGGCTCTGCAGCTTAG-3' \\
\hline & Reverse 5'-GCCGGTTACAGAACCACAC-3' \\
\hline \multirow[t]{2}{*}{$\mathrm{SOX} 2$} & Forward 5'-ACACCAATCCCATCCACACT-3' \\
\hline & Reverse 5'-GCAAACTTCCTGCAAAGCTC-3' \\
\hline \multirow[t]{2}{*}{ Nanog } & Forward 5'-CCTGATTCTTCCACCAGTCC-3' \\
\hline & Reverse 5'-TGCTATTCTTCGGCCAGTTG-3' \\
\hline \multirow[t]{2}{*}{ CD44 } & Forward 5'-TCACAGTGGAAGAAGAGAC-3' \\
\hline & Reverse 5'-CAT TG CATGTTGTCACT-3' \\
\hline \multirow[t]{2}{*}{ PDGF } & Forward 5'-CTCAGGCGAGATGACTTGTA-3' \\
\hline & Reverse 5'-CCACACCATCGTCCTCTAAT-3' \\
\hline \multirow[t]{2}{*}{ VEGF } & Forward 5'-CCTTGCTGCTCTACCTCCAC-3' \\
\hline & Reverse 5'-ATCTGCATGGTGATGTTGGA-3' \\
\hline \multirow[t]{2}{*}{ IL-8 } & Forward 5'-GCTCTGTGTGAAGGTGCAGTTT-3' \\
\hline & Reverse 5'-TTCTGTGTTGGCGCAGTGT-3' \\
\hline
\end{tabular}

Endothelial tube formation assay. The endothelial tubeformation assay was conducted according to the manufacturer's protocol (BD Biosciences, Franklin Lakes, NJ, USA). Matrigel $(50 \mu \mathrm{l})$ was added to each well of a 96-well plate and allowed to polymerize. EA.hy926 cells $\left(2 \times 10^{4}\right)$ plated on Matrigel was treated with the conditional medium of gastric cancer cells. After incubation for $12 \mathrm{~h}$ at $37^{\circ} \mathrm{C}$, the cells were viewed under a microscope and photographed. The number of formed tubes was counted under a microscope and five fields were randomly selected.

Immunohistochemistry. Immunohistochemistry was used to detect protein expression in tumor tissues sections. The antibodies used were anti- $\beta$-catenin (1:50; Cell Signaling Technology), CD31 (1:50; Bioworld Technology),
anti-E-cadherin, anti-N-cadherin (1:50; Santa Cruz Biotechnology), and anti-Ki67 (1:100; Boster, China). Ki67 staining is usually evaluated by counting positive cells of the total cell count (nucleus is stained by Ki67 antibody). Both cytosol and nucleus of the cells were positively stained for $\beta$-catenin protein.

Cell apoptosis assay. For cell apoptosis assay, a FITC Annexin V apoptosis detection kit (Vazyme, China) was used. SGC-7901 cells treated with GC-MSCs derived CM were trypsinized, washed in PBS and stained according to the manufacturer's instructions. The stained cells were analyzed by using flow cytometry (BD Accuri C6).

Animal model. Eighteen male BALB/c nu/nu mice (Laboratory Animal Center of Shanghai, Academy of Science, China) aged 4-6 weeks were randomly divided into 3 groups (6 mice/group). The animals were injected subcutaneously with untreated SGC-7901 cells (blank group), control GC-MSCs-CM treated SGC-7901 cells (sh-Ctrl CM group), and YAP knockdown GC-MSCs-CM treated SGC-7901 cells (sh-YAP CM group) $\left(1.5 \times 10^{6}\right.$ cells in $200 \mu \mathrm{l}$ PBS) into the right back side of mice. Tumor volumes were measured every 2 days using calipers according to the modified ellipsoidal formula: (length $\mathrm{x}$ width $\left.{ }^{2}\right) / 2$. The tumors were removed after injection for 28 days. The experiment protocols were approved by the Animal Use Committee of Jiangsu University.

Statistical analyses. All the data are presented as mean \pm standard deviation (SD). The statistically significant differences between groups were assessed by using analysis of variance (ANOVA) or t-test by Prism software (GraphPad, San Diego, USA). P-value $<0.05$ was considered statistically significant.

\section{Results}

YAP knockdown inhibits the proliferation of $G C$-MSCs. As one of the key components of tumor microenvironment (TME), MSCs have been found to play critical roles in tumor progression $(24,25)$. We have previously demonstrated that GC-MSCs promote gastric cancer growth more efficiently than adjacent non-cancerous gastric tissue-derived MSCs (GCN-MSCs) and bone marrow-derived MSCs (BM-MSCs) $(10,23)$. We hypothesized that YAP overexpression and activation is involved in the superior promoting role of GC-MSCs in gastric cancer growth. Thus, we compared YAP expression between GC-MSCs and BM-MSCs. The results of western blotting showed that the expression level of YAP in GC-MSCs was higher than that in BM-MSCs (Fig. 1A). Then, we used shRNA to knock down YAP in GC-MSCs and explored the effects of YAP knockdown on the proliferation of GC-MSCs (Fig. 1B). The results of colony formation assay showed that there were less colonies in YAP shRNA (shYAP) group than that in control group (Fig. 1C). The results of immunofluorescent staining revealed that there were more Ki67-positive cells in control group than that in shYAP group (Fig. 1D). The expression of PCNA was decreased in shYAP GC-MSCs compared to that in control GC-MSCs (Fig. 1B). Together, these results suggest that YAP knockdown inhibited the proliferation of GC-MSCs. 
A

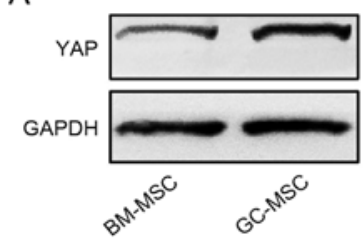

B

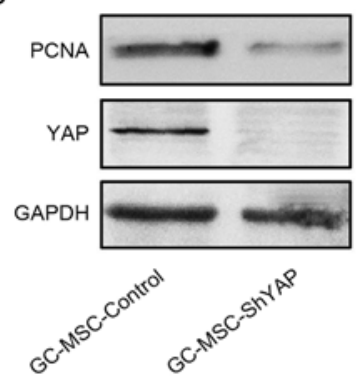

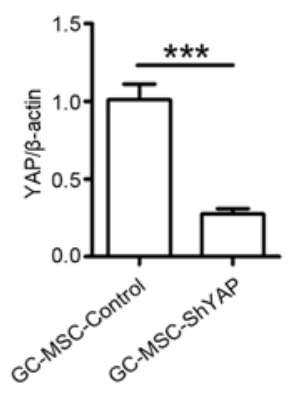

C

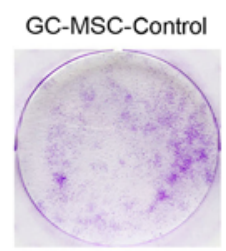

GC-MSC-ShYAP

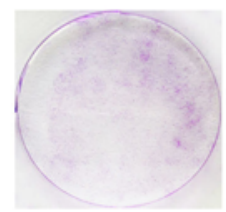

D
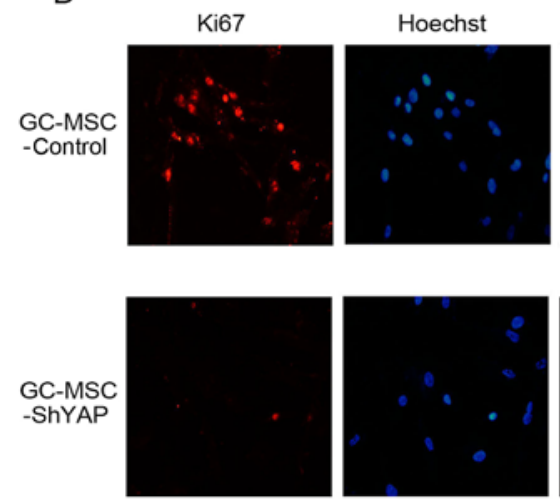

Merged
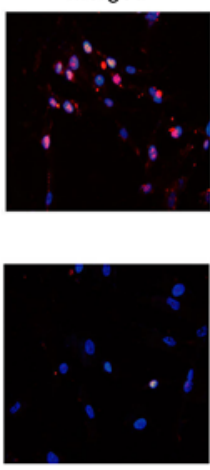

Figure 1. YAP knockdown inhibits the proliferation of GC-MSCs. (A) Western blotting for protein levels of YAP in BM-MSC and GC-MSC. (B) Western blotting for protein levels of PCNA, and YAP in GC-MSCs (control) and GC-MSCs (ShYAP). RT-PCR analysis of YAP expression in GC-MSCs (control) and GC-MSCs (ShYAP). ${ }^{* * *} \mathrm{P}<0.001$. (C) Colony formation assay in GC-MSCs (control) and GC-MSCs (ShYAP). (D) Immunofluorescence analysis revealed the expression level of Ki67 in GC-MSCs (control) and GC-MSCs (ShYAP). Magnification, x200; scale bar, $100 \mu \mathrm{m}$.

A

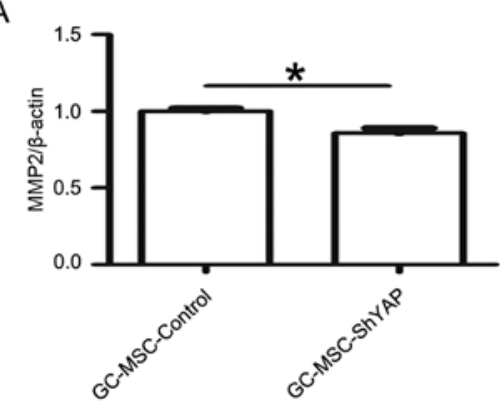

B
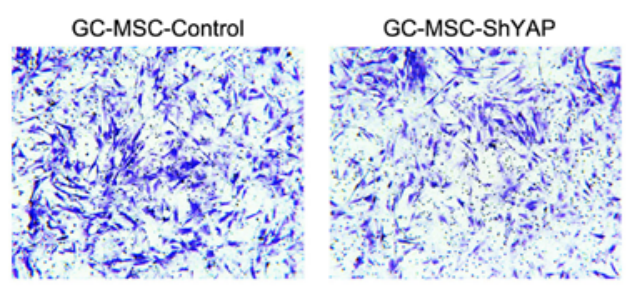

C
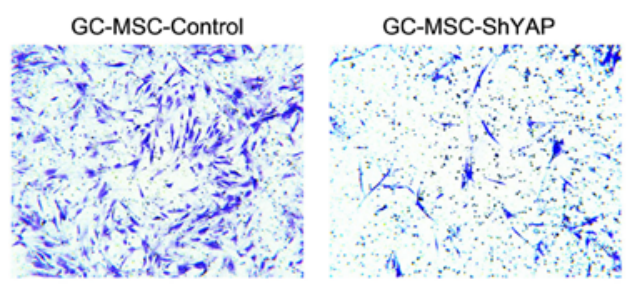
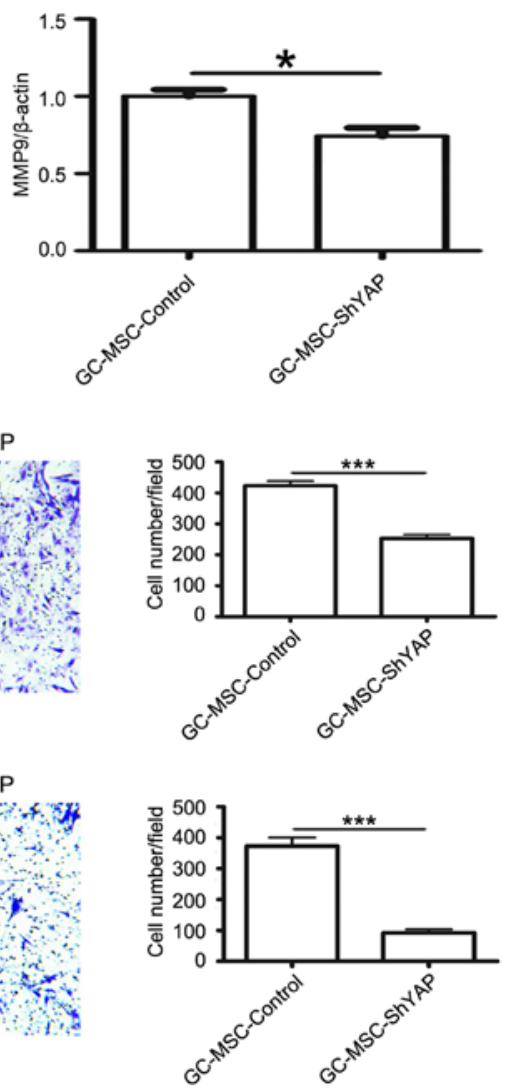

Figure 2. YAP knockdown inhibits the migration and invasion of GC-MSCs. (A) RT-PCR analysis of MMP2 and MMP9 expression in GC-MSCs (control) and GC-MSCs (ShYAP). "P<0.05. (B) Transwell migration assay histogram of migration cell number in GC-MSCs (control) and GC-MSCs (ShYAP). Magnification, x100; scale bar, $50 \mu \mathrm{m}$. (C) Transwell invasion assay histogram of invasion cell number in GC-MSCs (control) and GC-MSCs (ShYAP). Magnification, $\mathrm{x} 100$; scale bar, $50 \mu \mathrm{m} .{ }^{* * *} \mathrm{P}<0.001$. 
A

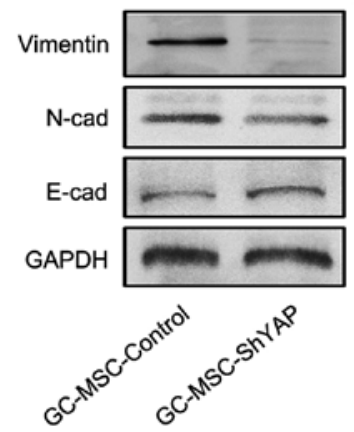

C

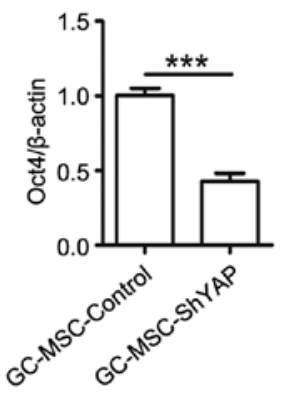

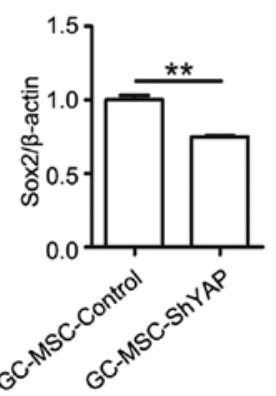

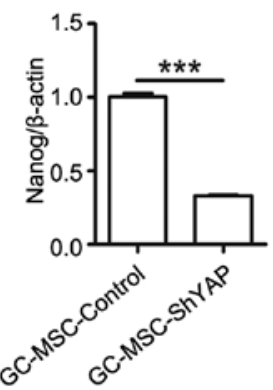

B
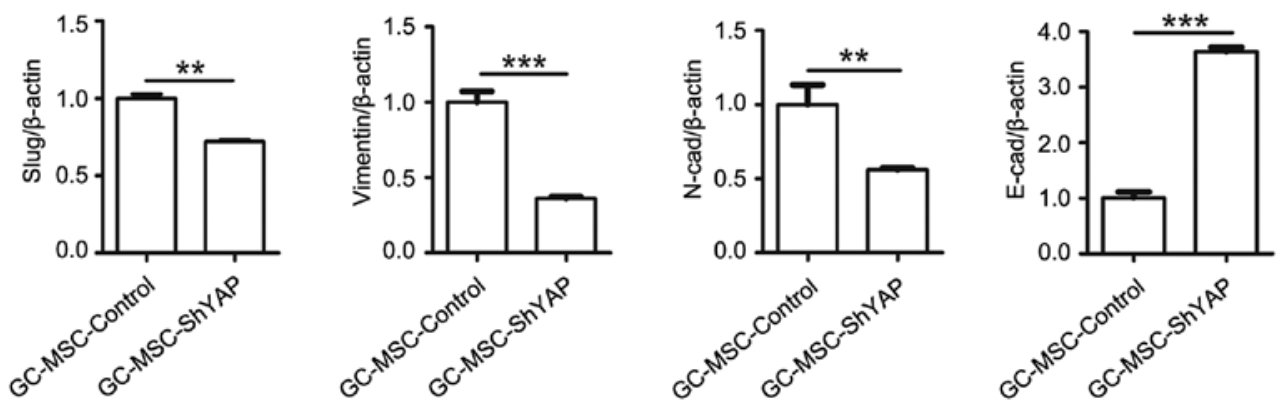

Figure 3. The knockdown of YAP suppresses EMT and stemness of GC-MSCs. (A) Western blotting for protein levels of Vimentin, N-cadherin and E-cadherin. (B) RT-PCR analysis of Slug, Vimentin, N-cadherin and E-cadherin expression in GC-MSCs (control) and GC-MSCs (ShYAP). ${ }^{* * *} \mathrm{P}<0.001$, ${ }^{* *} \mathrm{P}<0.01$. (C) RT-PCR analysis of Oct4, Sox2 and Nanog in GC-MSCs (control) and GC-MSCs (ShYAP). ${ }^{* * *} \mathrm{P}<0.001,{ }^{* *} \mathrm{P}<0.01$.

YAP knockdown inhibits the migration and invasion of $G C$-MSCs. We next investigated the effects of YAP knockdown on the migratory and invasive abilities of GC-MSCs. The expression of MMP2 and MMP9 was detected by using quantitative RT-PCR. YAP shRNA reduced the expression of MMP2 and MMP9 in GC-MSCs (Fig. 2A). Consistently, the number of cells migrating towards the lower surface of the chamber in the Transwell migration assay was lower in shYAP group than that in control group (Fig. 2B). Similar change was also observed in the matrigel invasion assay (Fig. 2C). Thus, these data suggest that YAP knockdown reduces the migratory and invasive abilities of GC-MSCs.

The knockdown of YAP suppresses EMT and stemness of $G C$-MSCs. We further investigated whether YAP affects mesenchymal-epithelial transition (MET) and stem cell properties of GC-MSCs. As shown in Fig. 3, YAP knockdown led to an increased expression of E-cadherin and a decreased expression of $\mathrm{N}$-cadherin, vimentin and slug in GC-MSCs (Fig. 3A and B).

To explore whether YAP maintains stem cell properties in GC-MSCs, we detected the expression of stem cell markers including Sox-2, Oct-4, and Nanog. As shown in Fig. 3C, YAP knockdown led to the reduced expression of Sox-2, Oct-4, and Nanog. Collectively, these results suggest that YAP may regulate the stem cell properties of GC-MSCs.

YAP knockdown in GC-MSCs reduced its promoting effect on gastric cancer cell growth in vitro. We further investigated whether YAP knockdown could affect the promoting role of GC-MSCs in the proliferation of gastric cancer cells. The results of cell colony formation assay showed that treatment with the conditioned media (CM) from control GC-MSCs increased the number of colonies of SGC-7901, HGC-27 and MGC-803 cells (Fig. 4A). However, when cultured with CM from shYAP GC-MSCs, the number of cell colonies was significantly decreased. The number of Ki67-positive cells was increased in SGC-7901 cells treated with CM from control GC-MSCs (sh-Ctrl CM group) but was decreased when treated with CM from shYAP GC-MSCs (sh-YAP CM group) (Fig. 4B). The results of western blotting showed that the expression of PCNA was increased in sh-Ctrl CM group but was decreased in sh-YAP CM group (Fig. 4C). There was no significant change in the expression of the anti-apoptotic protein $\mathrm{Bcl}-2$ and the pro-apoptotic protein Bax in gastric cancer cells when treated with $\mathrm{CM}$ from either control GC-MSCs or shYAP GC-MSCs (Fig. 4C). In addition, YAP knockdown had minimal effects on the apoptosis of SGC-7901 cells (Fig. 4D). In summary, YAP knockdown in GC-MSCs reduced its promoting role in the proliferation of gastric cancer cells.

YAP knockdown in GC-MSCs inhibits its promoting role in the migration and invasion of gastric cancer cells. We investigated the effects of YAP knockdown on the promoting role of GC-MSCs in gastric cancer cell migration and invasion. The gastric cancer cells in sh-YAP CM group exhibited lower migratory and invasive capacities than that in sh-Ctrl CM grou (Fig. 5A-D). We further investigated whether YAP knockdown in GC-MSCs affected its inducing effect on the EMT of gastric cancer cells. Sh-YAP CM group showed an increased expression of E-cadherin and a reduced expression 
A

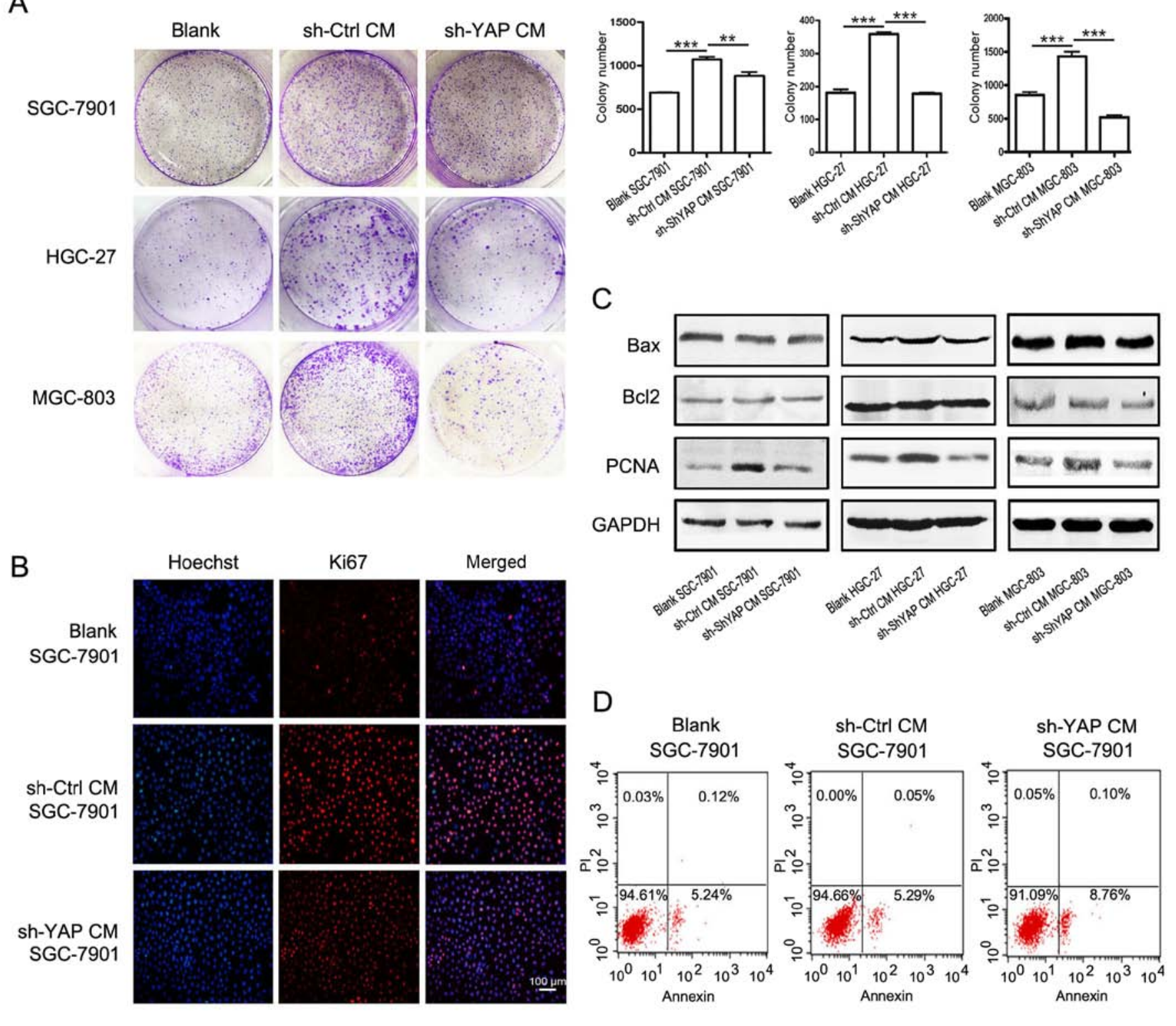

Figure 4. YAP knockdown in GC-MSC inhibits its promoting effect on the growth of gastric cancer cells in vitro. (A) Colony formation assay was performed to analyze the proliferation ability of gastric cancer cells (SGC-7901, HGC-27 and MGC-803) with different treatments. The colony number was counted. Blank gastric cancer cells, untreated gastric cancer cells; sh-Ctrl CM group, gastric cancer cells were treated with the $72 \mathrm{~h}$ conditioned medium of GC-MSCs transfected with control lentiviral; sh-YAP CM group gastric cancer cells, gastric cancer cells were treated with the $72 \mathrm{~h}$ conditioned medium of GC-MSCs transfected with ShYAP lentiviral. ${ }^{* *} \mathrm{P}<0.01,{ }^{* * *} \mathrm{P}<0.001$. (B) Immunofluorescence analysis revealed that the expression level of Ki67 in blank group, sh-Ctrl CM group and sh-YAP CM group. Magnification, x200; scale bar, $100 \mu \mathrm{m}$. (C) Western blotting for protein levels of Bax, Bcl2 and PCNA. (D) The apoptosis of gastric cancer cells was analyzed by using flow cytometry.

of Slug, Vimentin and N-cadherin compared with sh-Ctrl CM group (Fig. 5E and F). These results indicate that YAP knockdown in GC-MSCs inhibited its promoting role in the migration and invasion of gastric cancer cells.

YAP knockdown in GC-MSCs inhibits its promoting role in the pro-angiogenic ability of gastric cancer cells. Angiogenesis is considered as a critical step for cancer development and progression. MSCs can favor the formation of tumor blood vessels and thus promote tumor growth and metastasis (26). As shown in Fig. 6, the incubation with $\mathrm{CM}$ from shYAP GC-MSCs dramatically decreased the expression of VEGF, PDGF, and IL-8 in gastric cancer cells compared to incubation with CM from control GC-MSCs (Fig. 6A). The results of tube formation assay demonstrated that the supernatant of sh-YAP CM group had reduced ability to promote endothelial cell tube formation than that of sh-Ctrl CM group (Fig. 6B). Endothelial cell migration is critical for angiogenesis. The results of Transwell migration assay showed that the CM from sh-YAP CM group had decreased ability to promote the migration of endothelial cells compared to the $\mathrm{CM}$ from sh-Ctrl CM group (Fig. 6C). Taken together, these results reveal that YAP knockdown in GC-MSCs inhibits its promoting role in the pro-angiogenic ability of gastric cancer cells.

YAP knockdown in GC-MSCs reduced its promoting effect on the activation of $\beta$-catenin in gastric cancer cells. $\beta$-catenin is 

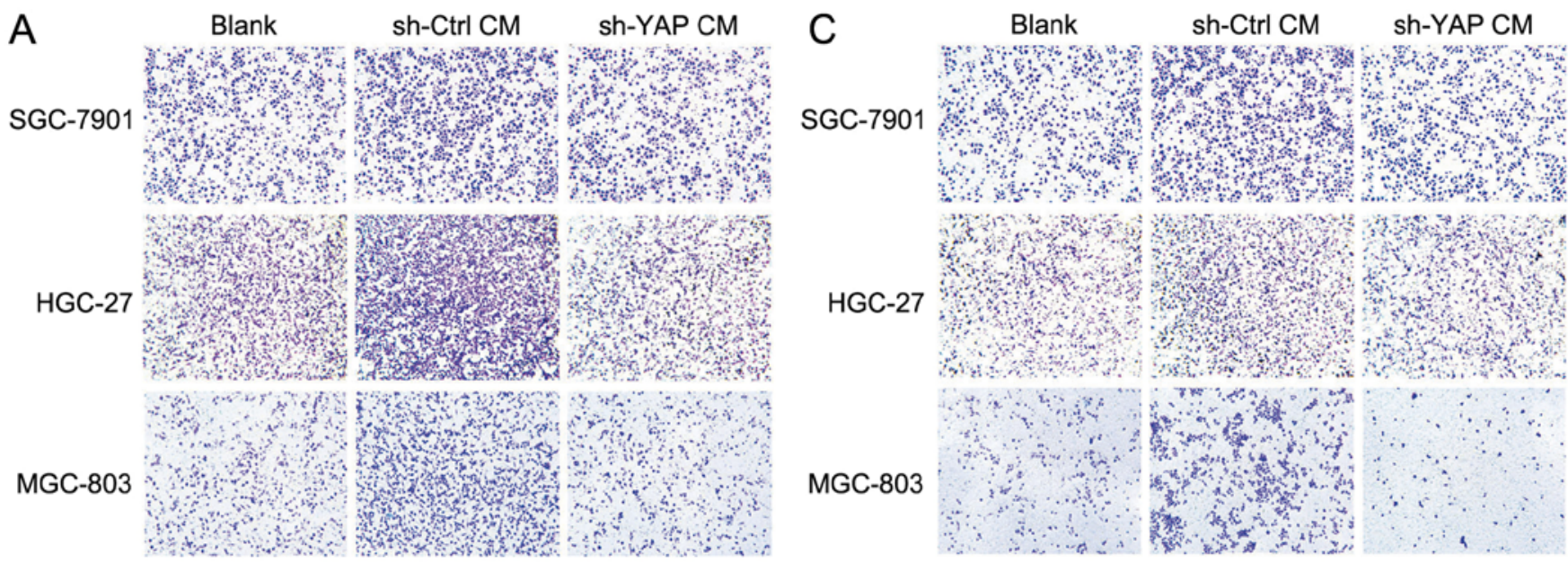

B
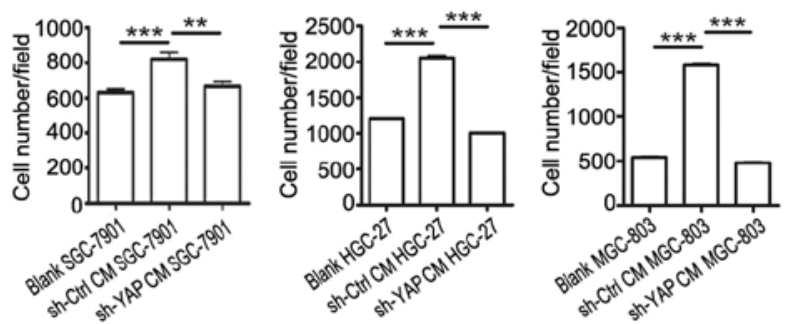

D
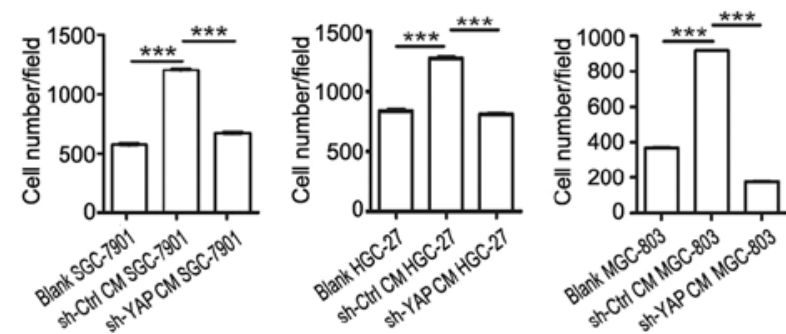

$\mathrm{F}$
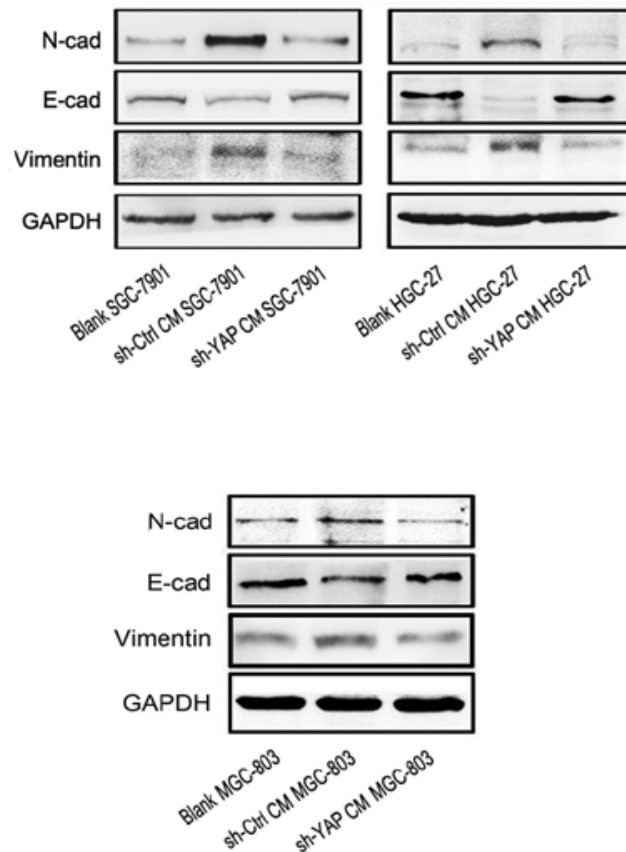

Figure 5. YAP knockdown in GC-MSCs inhibits its promoting role in the migration and invasion of gastric cancer cells. (A) Transwell migration assay for blank group, sh-Ctrl CM group and sh-YAP CM group gastric cancer cells. (B) Cells that migrated to the bottom were counted. ${ }^{* * *} \mathrm{P}<0.001,{ }^{* * *} \mathrm{P}<0.01$. (C) Matrigel invasion assay for blank group, sh-Ctrl CM group and sh-YAP CM group gastric cancer cells. (D) Cells that invaded to the bottom were counted. ${ }^{* * *} \mathrm{P}<0.001$. (E) RT-PCR analysis of Slug, Vimentin, N-cadherin, E-cadherin and Slug expression in blank group, sh-Ctrl CM group and sh-YAP CM group gastric cancer cells. ${ }^{* * *} \mathrm{P}<0.001,{ }^{* *} \mathrm{P}<0.01,{ }^{*} \mathrm{P}<0.05$. (F) Western blotting for protein levels of $\mathrm{N}$-cadherin, E-cadherin and Vimentin in blank group, sh-Ctrl $\mathrm{CM}$ group and sh-YAP CM group gastric cancer cells.

an important pathway in gastric carcinogenesis (27-31). MSCs enhanced the activation of $\beta$-catenin signaling in cancer. We then examined the role of YAP in MSC-mediated regulation of $\beta$-catenin signaling in gastric cancer cells. As shown in
Fig. 7A, the expression of $\beta$-catenin was lower in sh-YAP CM group compared to that in sh-Ctrl CM group. Moreover, the expression of $\beta$-catenin downstream genes including cyclin D and CD44 was lower in sh-YAP CM group than that in 
A
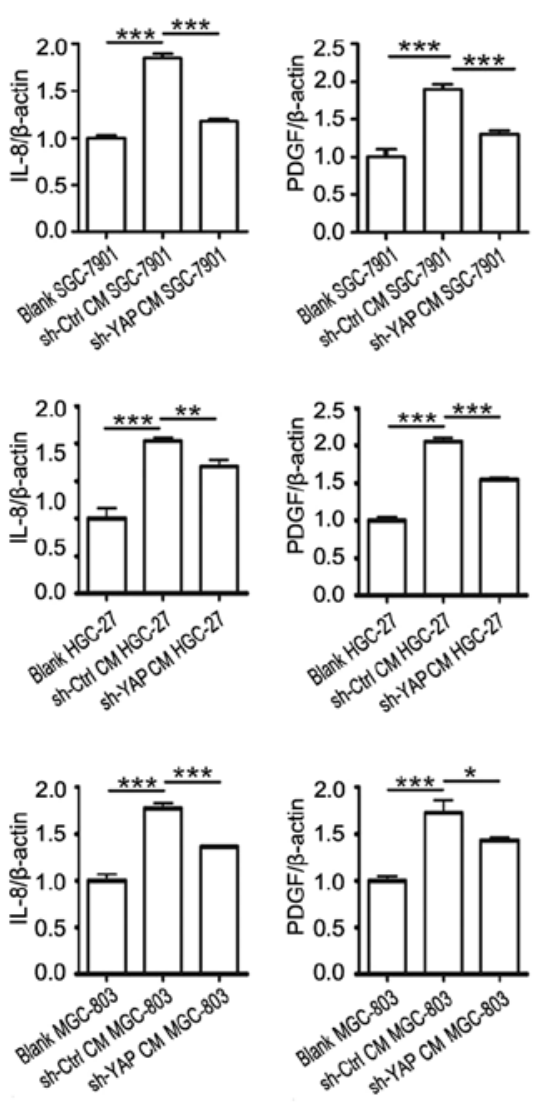

B
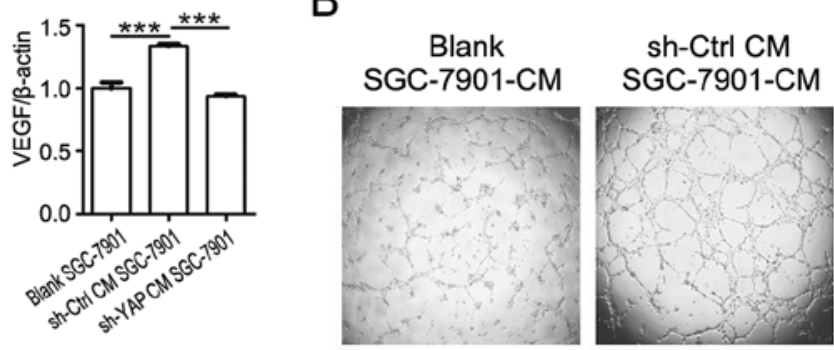

Sh-YAP CM SGC-7901-CM
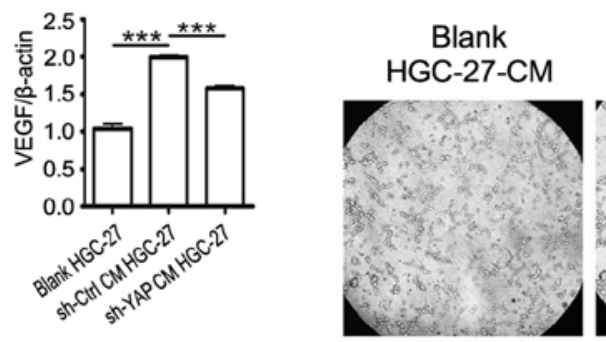

sh-Ctrl CM
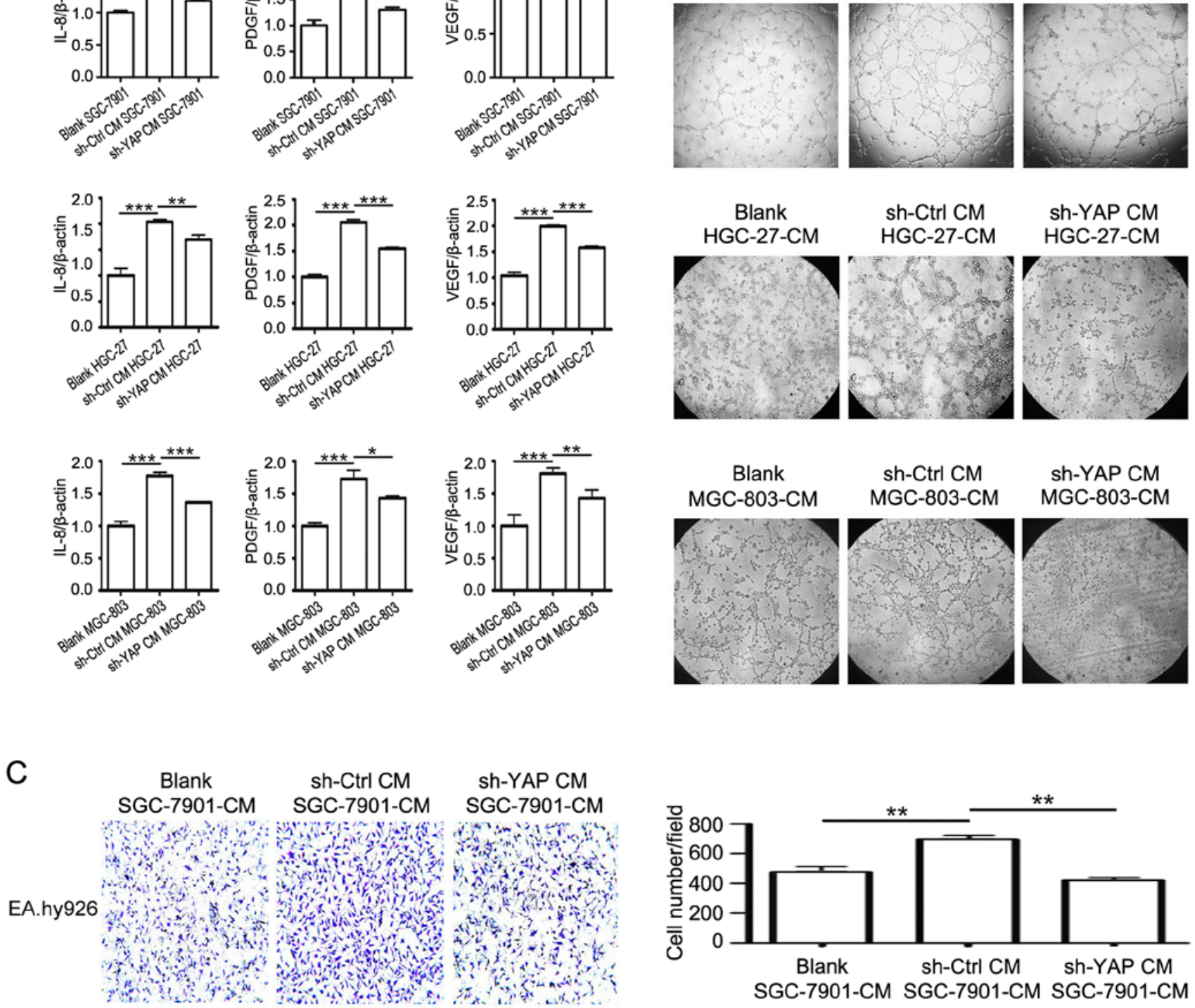

Figure 6. YAP knockdown in GC-MSCs inhibits its promoting role in the pro-angiogenic ability of gastric cancer cells. (A) RT-PCR analyses of IL-8, PDGF and VEGF expression in blank group, sh-Ctrl CM group and sh-YAP CM group gastric cancer cells. ${ }^{* * * *} \mathrm{P}<0.001,{ }^{* * *} \mathrm{P}<0.01,{ }^{*} \mathrm{P}<0.05$. (B) The endothelial tubeformation assay for blank group, sh-Ctrl CM group and sh-YAP CM group gastric cancer cells. (C) EA.hy926 cells were separately treated with conditioned medium from blank group, sh-Ctrl CM group and sh-YAP CM group gastric cancer cells for $12 \mathrm{~h}$. Transwell migration assay was performed to analyze the migratory ability of the cells. Cells that migrated to the bottom were counted. Magnification, $\mathrm{x} 100$; scale bar, $50 \mu \mathrm{m} .{ }^{* *} \mathrm{P}<0.01$.

sh-Ctrl CM group (Fig. 7B). Collectively, these results suggest that YAP knockdown in GC-MSCs reversed their activating role in $\beta$-catenin signaling in gastric cancer cells.

YAP knockdown in GC-MSCs inhibits its promoting role in gastric cancer growth in vivo. To confirm the in vitro results, SGC-7901 cells treated with CM from control and shYAP GC-MSCs were used to establish mouse xenograft tumor models. The images of xenograft tumors are shown in Fig. 8A. The tumors in sh-YAP CM group grew slower and were smaller, and the average tumor weight was lower than that in sh-Ctrl CM group (Fig. 8B). The expression of Ki67 and $\beta$-catenin in tumor tissues was determined by using immunohistochemistry. We found that the percentage of Ki67-positive cells was $24.0 \%$ in sh-YAP CM group and was $92.1 \%$ in
sh-Ctrl CM group. The expression of $\beta$-catenin in the nucleus was stronger in sh-Ctrl CM group than that in sh-YAP CM group (Fig. 8C). The decreased expression of E-cadherin observed in sh-Ctrl CM group were reversed in sh-YAP CM group (Fig. 8C). Moreover, CD31 expression in sh-YAP CM group was significantly lower than that in sh-Ctrl CM group (Fig. 8D). Taken together, these results suggest that YAP knockdown in GC-MSCs reversed its promoting role in gastric cancer growth in vivo.

\section{Discussion}

Over the past decades, the relation between MSCs and tumor has attracted increasing attention $(8,25,32)$. Although the previous studies have shown an important role of MSCs 
A

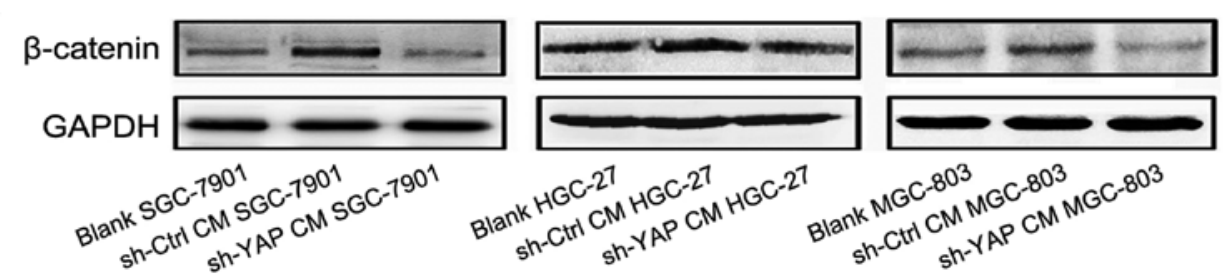

B
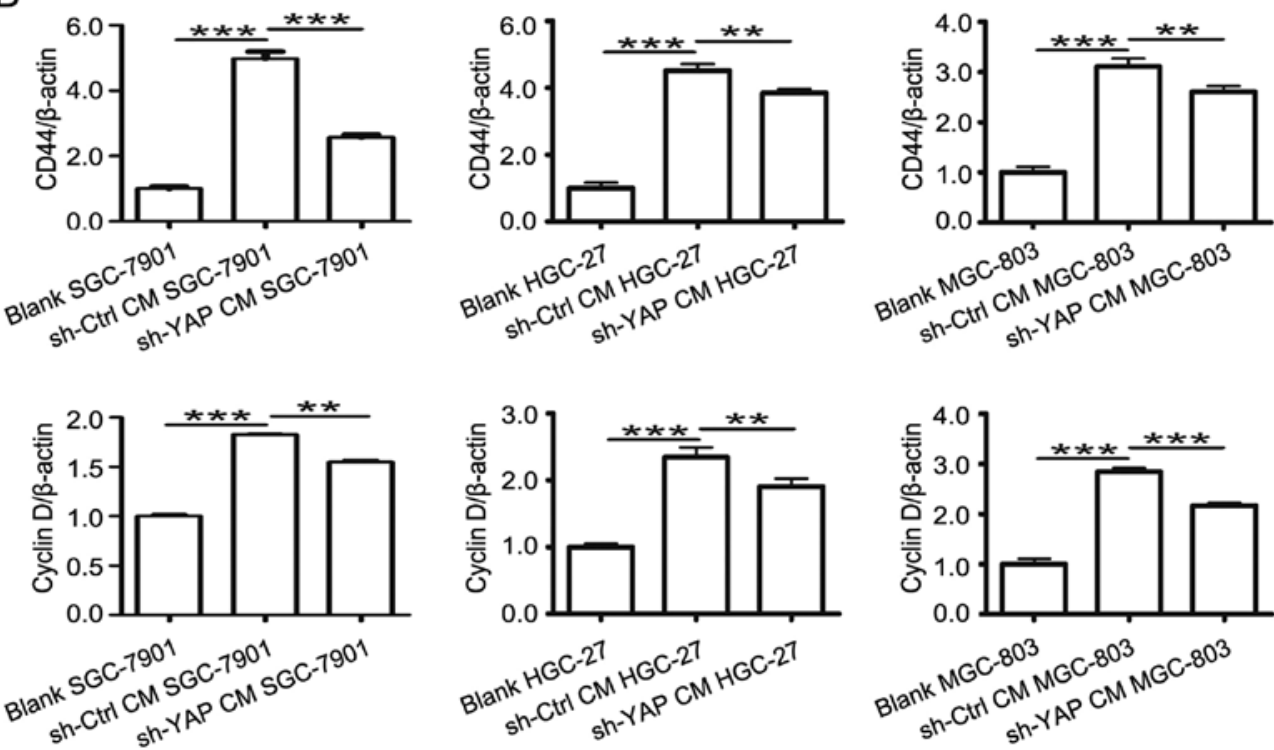

Figure 7. YAP knockdown in GC-MSCs reduced its promoting effect on the activation of $\beta$-catenin in gastric cancer cells. (A) Western blotting for protein levels of $\beta$-catenin in blank group, sh-Ctrl CM group and sh-YAP CM group gastric cancer cells. (B) RT-PCR analyses of CD44 and cyclin D expression in blank group, sh-Ctrl CM group and sh-YAP CM group gastric cancer cells. ${ }^{* * *} \mathrm{P}<0.001,{ }^{* *} \mathrm{P}<0.01,{ }^{*} \mathrm{P}<0.05$.

in cancer (33-35), the detailed mechanisms responsible for the regulation of tumor-resident MSCs are not clear. The Hippo/YAP pathway has recently been reported to play important roles in human cancers (36-38). As a critical component of Hippo pathways, YAP could exert oncogenic activities with its paralog transcriptional co-activator with a PDZ-binding motif (TAZ) (39). The expression of YAP in gastric cancer tissues is closely associated with poorer overall survival of patients. RUNX3 is reported to function as a tumor suppressor by downregulating YAP in the progression of cancer (40). Moreover, VGLL4 could inhibit the expression of YAP, and a peptide is found to act as a YAP antagonist therapy against gastric cancer by mimicking VGLL4 function (41).

The interaction between MSCs and tumor cells is critical for tumor progression $(21,42)$. GC-MSCs enhanced the proliferation and migration of gastric cancer cells as well as facilitate tumor angiogenesis (10). In this study, we identified that YAP signaling was critical for the promoting roles of GC-MSCs in gastric cancer progression. We reported that YAP knockdown led to the inhibition of the growth, migration, and invasion of GC-MSCs, indicating that YAP plays an important role in the phenotype and function of GC-MSCs.

The oncogenic roles of YAP in cancer has recently been a research focus (43-45). In this study, we reported that gastric cancer cells grow slower when incubated with CM from YAP knockdown GC-MSCs. However, YAP knockdown in GC-MSCs have no effects on the apoptosis of gastric cancer cells. It was reported that YAP could promote the growth of cholangiocarcinoma by interacting with TEAD transcription factors (49). Sun and colleagues found that YAP could enhance the proliferation, migration, and invasion of gastric cancer cells in vitro and in vivo (16). The decreased YAP signaling inhibited tumor growth and metastasis by reducing the expression of PCNA, MMP-2, MMP-9, and cyclin D1 (45). In the present study, we found that YAP knockdown in GC-MSCs abrogated its promoting roles in gastric cancer cell proliferation, migration, and invasion, indicating an important role of YAP signaling in the tumor-promoting effect of GC-MSCs in gastric cancer. Moreover, YAP could also promote angiogenesis in human cancer (46). We observed that endothelial cells exposed to the supernatant from sh-YAP CM-treated gastric cancer cells showed decreased tube formation and migration abilities, which may be associated with the decreased expression of pro-angiogenic factors including VEGF, PDGF, and IL-8 in gastric cancer cells. These findings suggest a potent role of YAP in GC-MSCs in regulating tumor angiogenesis. Metastasis is associated with increased cell migration and invasion. The $\beta$-catenin pathway is reported to affect the migration and invasion of cancer cells (47). In our study, YAP knockdown in GC-MSCs inhibited its promoting role in the activation of $\beta$-catenin and the migration and invasion of gastric cancer cells. Thus, YAP signaling in GC-MSCs may promote gastric cancer metastasis through an indirect activation of $\beta$-catenin pathway in gastric cancer cells. 
A

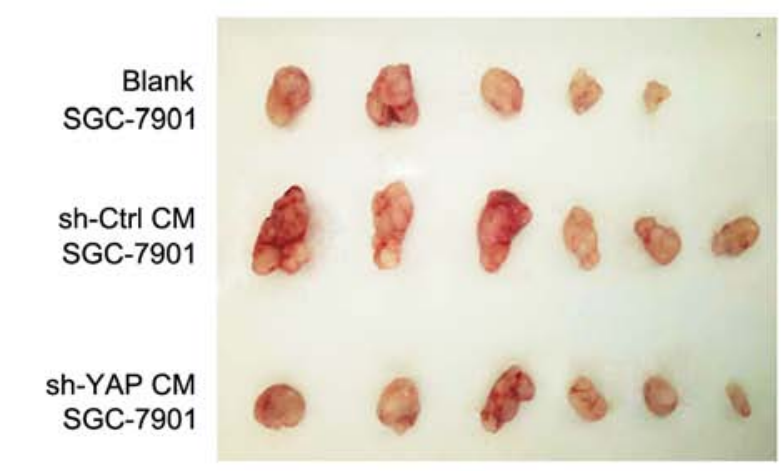

C $\beta$-catenin

\section{sh-Ctrl CM} SGC-7901

Sh-YAP CM SGC-7901

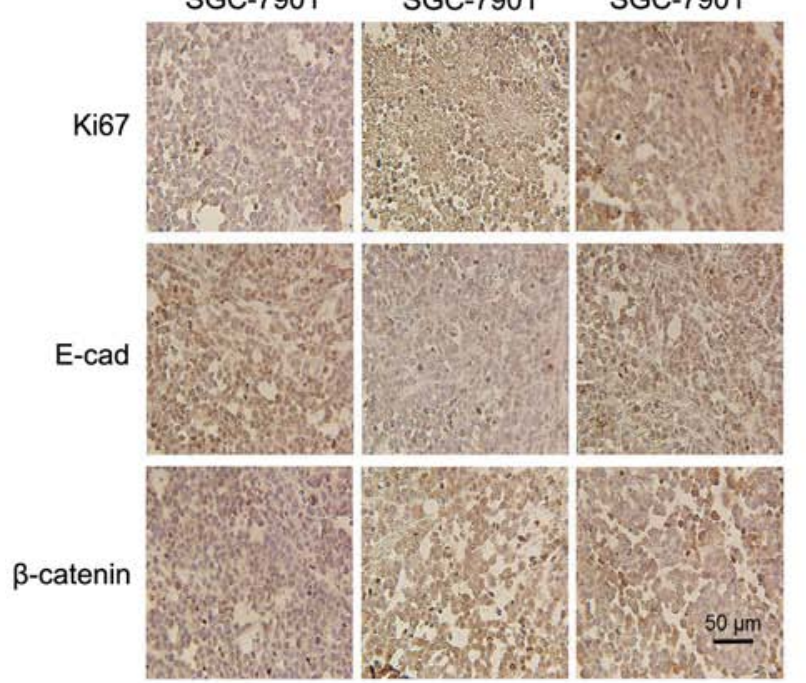

B
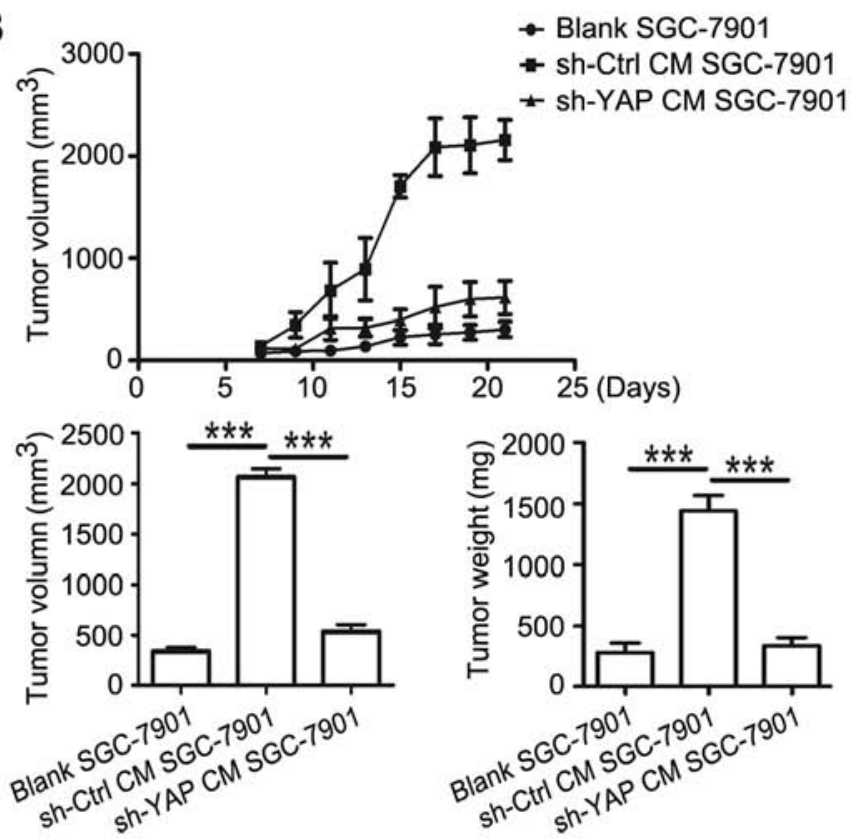

D
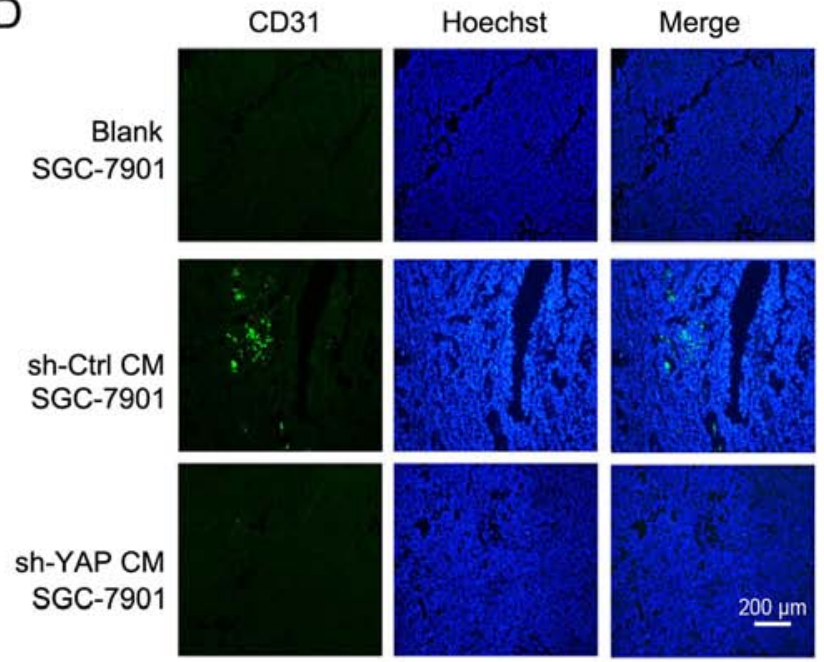

Figure 8. YAP knockdown in GC-MSCs inhibits its promoting role in gastric cancer growth in vivo. (A) Tumor tissues were removed and photographed at 28 days after tumor cell inoculation. (B) Tumor volume and weight in blank, sh-Ctrl CM, and sh-YAP CM groups. ${ }^{* * *} \mathrm{P}<0.001$. (C) Ki67, E-cadherin and $\beta$-catenin expression were determined by using IHC staining in tumor tissues from blank, sh-Ctrl CM and sh-YAP CM groups. The percentage of Ki67-positive cells in the total cells were counted. The cytosol was positively stained for E-cadherin. Both cytosol and nucleus of the cells were positively stained for $\beta$-catenin protein. Magnification, $\mathrm{x} 400$; scale bar, $50 \mu \mathrm{m}$. (D) Representative immunofluorescence images of CD31 expression in tumors from blank, sh-Ctrl CM and sh-YAP CM groups. Magnification, x100; scale bar, $200 \mu \mathrm{m}$.

The $\beta$-catenin pathway contributes to cancer progression by regulating the proliferation, invasion, and metastasis of cancer cells (47-50). Our results revealed that the increased expression of $\beta$-catenin in sh-Ctrl CM group was abrogated in the sh-YAP CM group. In addition, the expression of $\beta$-catenin downstream genes CD44 and cyclin D1 was also decreased in sh-YAP CM group compared to sh-Ctrl CM group. These findings suggest that YAP signaling modulates GC-MSC-mediated activation of $\beta$-catenin in gastric cancer cells. We have recently reported that YAP critically regulates the activity of $\beta$-catenin (51). YAP knockdown may affect the components of CM from GC-MSCs, which thus abrogates the activation of $\beta$-catenin signaling in tumor cells. However, the exact factors responsible for this role need to be identified in future studies.
In conclusion, we demonstrated that YAP knockdown in GC-MSCs not only inhibits their proliferation, migration and invasion, but also suppresses their promoting roles in the proliferation, migration, invasion and pro-angiogenesis of gastric cancer cells in vitro and in vivo. Disturbing the expression of YAP in GC-MSCs inhibits its derived CM-induced activation of $\beta$-catenin in gastric cancer cells. In conclusion, YAP expression in GC-MSCs plays an important role in promoting gastric cancer progression, which may provide a novel avenue for gastric cancer therapy.

\section{Acknowledgements}

This study was supported by the Major Research Plan of the National Natural Science Foundation of China (grant 
no.91129718), the National Natural Science Foundation of China (grant nos. 81572075, 81672416 and 816702883), the Project of Major Research and Development, Jiangsu Province (grant no. BE2015667), the Doctoral Program Foundation of China (grant nos. 2016M591791 and 2016M591792), the Doctoral Program Foundation, Jiangsu Province (grant no. 1501067C), Jiangsu Province for Outstanding Sci-Tech Innovation Team in Colleges and Universities (grant no. SJK2013-10), and Project Funded by the Priority Academic Program Development of Jiangsu Higher Education Institutions.

\section{References}

1. Tan YK and Fielding JW: Early diagnosis of early gastric cancer. Eur J Gastroenterol Hepatol 18: 821-829, 2006.

2. Bertuccio P, Chatenoud L, Levi F, Praud D, Ferlay J, Negri E, Malvezzi M and La Vecchia C: Recent patterns in gastric cancer: A global overview. Int J Cancer 125: 666-673, 2009.

3. Jemal A, Bray F, Center MM, Ferlay J, Ward E and Forman D: Global cancer statistics. CA Cancer J Clin 61: 69-90, 2011.

4. Pornsuksiri K, Chewatanakornkul S, Kanngurn S, Maneechay W, Chaiyapan W and Sangkhathat S: Clinical outcomes of gastrointestinal stromal tumor in southern Thailand. World J Gastrointest Oncol 4: 216-222, 2012

5. Yu B and Xie J: Identifying therapeutic targets in gastric cancer: The current status and future direction. Acta Biochim Biophys Sin (Shanghai) 48: 90-96, 2016.

6. Kasashima H, Yashiro M, Nakamae H, Kitayama K, Masuda G, Kinoshita H, Fukuoka T, Hasegawa T, Nakane T, Hino M, et al: CXCL1-chemokine (C-X-C motif) receptor 2 signaling stimulates the recruitment of bone marrow-derived mesenchymal cells into diffuse-type gastric cancer stroma. Am J Pathol 186: 3028-3039, 2016.

7. Wu L, Zhang X, Zhang B, Shi H, Yuan X, Sun Y, Pan Z, Qian H and $\mathrm{Xu} \mathrm{W}$ : Exosomes derived from gastric cancer cells activate NF- $\kappa \mathrm{B}$ pathway in macrophages to promote cancer progression. Tumour Biol 37: 12169-12180, 2016.

8. Huang F, Wang M, Yang T, Cai J, Zhang Q, Sun Z, Wu X, Zhang X, Zhu W, Qian H, et al: Gastric cancer-derived MSC-secreted PDGF-DD promotes gastric cancer progression. J Cancer Res Clin Oncol 140: 1835-1848, 2014.

9. Wang M, Zhao C, Shi H, Zhang B, Zhang L, Zhang X, Wang S, Wu X, Yang T, Huang F, et al: Deregulated microRNAs in gastric cancer tissue-derived mesenchymal stem cells: Novel biomarkers and a mechanism for gastric cancer. Br J Cancer 110: 1199-1210, 2014.

10. Li W, Zhou Y, Yang J, Zhang X, Zhang H, Zhang T, Zhao S, Zheng P, Huo J and Wu H: Gastric cancer-derived mesenchymal stem cells prompt gastric cancer progression through secretion of interleukin-8. J Exp Clin Cancer Res 34: 52, 2015.

11. Yu FX, Zhao B and Guan KL: Hippo pathway in organ size control, tissue homeostasis, and cancer. Cell 163: 811-828, 2015.

12. Harvey KF, Zhang X and Thomas DM: The Hippo pathway and human cancer. Nat Rev Cancer 13: 246-257, 2013.

13. Moroishi T, Hansen CG and Guan KL: The emerging roles of YAP and TAZ in cancer. Nat Rev Cancer 15: 73-79, 2015.

14. Hua G, Lv X, He C, Remmenga SW, Rodabough KJ, Dong J, Yang L, Lele SM, Yang P, Zhou J, et al: YAP induces high-grade serous carcinoma in fallopian tube secretory epithelial cells. Oncogene 35: 2247-2265, 2016.

15. Zhang J, Xu ZP, Yang YC, Zhu JS, Zhou Z and Chen WX: Expression of Yes-associated protein in gastric adenocarcinoma and inhibitory effects of its knockdown on gastric cancer cell proliferation and metastasis. Int J Immunopathol Pharmacol 25: 583-590, 2012

16. Sun D, Li X, He Y, Li W, Wang Y, Wang H, Jiang S and Xin Y: YAP1 enhances cell proliferation, migration, and invasion of gastric cancer in vitro and in vivo. Oncotarget 7: 81062-81076, 2016.

17. Calvo F, Ege N, Grande-Garcia A, Hooper S, Jenkins RP, Chaudhry SI, Harrington K, Williamson P, Moeendarbary E, Charras G, et al: Mechanotransduction and YAP-dependent matrix remodelling is required for the generation and maintenance of cancer-associated fibroblasts. Nat Cell Biol 15: 637-646, 2013.
18. Zhang J, Ji JY, Yu M, Overholtzer M, Smolen GA, Wang R, Brugge JS, Dyson NJ and Haber DA: YAP-dependent induction of amphiregulin identifies a non-cell-autonomous component of the Hippo pathway. Nat Cell Biol 11: 1444-1450, 2009.

19. Fujii M, Toyoda T, Nakanishi H, Yatabe Y, Sato A, Matsudaira Y, Ito $\mathrm{H}$, Murakami $\mathrm{H}$, Kondo $\mathrm{Y}$, Kondo E, et al: TGF- $\beta$ synergizes with defects in the Hippo pathway to stimulate human malignant mesothelioma growth. J Exp Med 209: 479-494, 2012.

20. Mo JS, Park HW and Guan KL: The Hippo signaling pathway in stem cell biology and cancer. EMBO Rep 15: 642-656, 2014.

21. Zhang T, Lee YW, Rui YF, Cheng TY, Jiang XH and Li G: Bone marrow-derived mesenchymal stem cells promote growth and angiogenesis of breast and prostate tumors. Stem Cell Res Ther 4: 70, 2013.

22. Cao H, Xu W, Qian H, Zhu W, Yan Y, Zhou H, Zhang X, Xu X, Li J, Chen Z, et al: Mesenchymal stem cell-like cells derived from human gastric cancer tissues. Cancer Lett 274: 61-71, 2009.

23. Xu X, Zhang X, Wang S, Qian H, Zhu W, Cao H, Wang M, Chen $\mathrm{Y}$ and $\mathrm{Xu} \mathrm{W}$ : Isolation and comparison of mesenchymal stem-like cells from human gastric cancer and adjacent noncancerous tissues. J Cancer Res Clin Oncol 137: 495-504, 2011.

24. Bergfeld SA and DeClerck YA: Bone marrow-derived mesenchymal stem cells and the tumor microenvironment. Cancer Metastasis Rev 29: 249-261, 2010.

25. Barcellos-de-Souza P, Gori V, Bambi F and Chiarugi P: Tumor microenvironment: Bone marrow-mesenchymal stem cells as key players. Biochim Biophys Acta 1836: 321-335, 2013.

26. Li GC, Zhang HW, Zhao QC, Sun LI, Yang JJ, Hong L, Feng F and Cai L: Mesenchymal stem cells promote tumor angiogenesis via the action of transforming growth factor $\beta 1$. Oncol Lett 11: 1089-1094, 2016.

27. Su YJ, Lin WH, Chang YW, Wei KC, Liang CL, Chen SC and Lee JL: Polarized cell migration induces cancer type-specific CD133/integrin/Src/Akt/GSK3 $\beta / \beta$-catenin signaling required for maintenance of cancer stem cell properties. Oncotarget 6 : 38029-38045, 2015.

28. Chen MJ, Wu DW, Wang YC, Chen CY and Lee H: PAK1 confers chemoresistance and poor outcome in non-small cell lung cancer via $\beta$-catenin-mediated stemness. Sci Rep 6: 34933, 2016.

29. Lettini G, Sisinni L, Condelli V, Matassa DS, Simeon V, Maddalena F, Gemei M, Lopes E, Vita G, Del Vecchio L, et al: TRAP1 regulates stemness through $\mathrm{Wnt} / \beta$-catenin pathway in human colorectal carcinoma. Cell Death Differ 23: 1792-1803, 2016.

30. Liu D, Du L, Chen D, Ye Z, Duan H, Tu T, Feng J, Yang Y, Chen Q and Yan X: Reduced CD146 expression promotes tumorigenesis and cancer stemness in colorectal cancer through activating Wnt/ $\beta$-catenin signaling. Oncotarget 7: 40704-40718, 2016.

31. Zhang X, Xu J, Jiang T, Liu G, Wang D and Lu Y: MicroRNA-195 suppresses colorectal cancer cells proliferation via targeting FGF2 and regulating Wnt/ $\beta$-catenin pathway. Am J Cancer Res 6: 2631-2640, 2016.

32. Gabrielyan A, Knaak S, Gelinsky M, Arnhold S and RösenWolff A: Hypoxia-conditioned media allows species-specific attraction of bone marrow stromal cells without need for recombinant proteins. BMC Vet Res 10: 56, 2014.

33. Bergfeld SA, Blavier L and DeClerck YA: Bone marrow-derived mesenchymal stromal cells promote survival and drug resistance in tumor cells. Mol Cancer Ther 13: 962-975, 2014.

34. Han I, Yun M, Kim EO, Kim B, Jung MH and Kim SH: Umbilical cord tissue-derived mesenchymal stem cells induce apoptosis in PC-3 prostate cancer cells through activation of JNK and downregulation of PI3K/AKT signaling. Stem Cell Res Ther 5: 54, 2014.

35. Zhu M, Wang M, Yang F, Tian Y, Cai J, Yang H, Fu H, Mao F, Zhu W, Qian H, et al: miR-155-5p inhibition promotes the transition of bone marrow mesenchymal stem cells to gastric cancer tissue derived MSC-like cells via NF- $\mathrm{B}$ p 65 activation. Oncotarget 7: 16567-16580, 2016.

36. Steinhardt AA, Gayyed MF, Klein AP, Dong J, Maitra A, Pan D, Montgomery EA and Anders RA: Expression of Yes-associated protein in common solid tumors. Hum Pathol 39: 1582-1589, 2008.

37. Zhao B, Li L, Lei Q and Guan KL: The Hippo-YAP pathway in organ size control and tumorigenesis: An updated version. Genes Dev 24: 862-874, 2010.

38. Azzolin L, Panciera T, Soligo S, Enzo E, Bicciato S, Dupont S, Bresolin S, Frasson C, Basso G, Guzzardo V, et al: YAP/TAZ incorporation in the $\beta$-catenin destruction complex orchestrates the Wnt response. Cell 158: 157-170, 2014. 
39. Zuo QF, Zhang R, Li BS, Zhao YL, Zhuang Y, Yu T, Gong L, Li S, Xiao B and Zou QM: MicroRNA-141 inhibits tumor growth and metastasis in gastric cancer by directly targeting transcriptional co-activator with PDZ-binding motif, TAZ. Cell Death Dis 6: e1623, 2015.

40. Qiao Y, Lin SJ, Chen Y, Voon DC, Zhu F, Chuang LS, Wang T, Tan P, Lee SC, Yeoh KG, et al: RUNX3 is a novel negative regulator of oncogenic TEAD-YAP complex in gastric cancer. Oncogene 35: 2664-2674, 2016.

41. Jiao S, Wang H, Shi Z, Dong A, Zhang W, Song X, He F, Wang Y, Zhang $\mathrm{Z}$, Wang W, et al: A peptide mimicking VGLL4 function acts as a YAP antagonist therapy against gastric cancer. Cancer Cell 25: 166-180, 2014.

42. Liu C, Liu Y, Xu XX, Guo X, Sun GW and Ma XJ: Mesenchymal stem cells enhance the metastasis of 3D-cultured hepatocellular carcinoma cells. BMC Cancer 16: 566, 2016.

43. Ehmer U and Sage J: Control of proliferation and cancer growth by the Hippo signaling pathway. Mol Cancer Res 14: 127-140, 2016.

44. Yagi H, Asanoma K, Ohgami T, Ichinoe A, Sonoda K and Kato K: GEP oncogene promotes cell proliferation through YAP activation in ovarian cancer. Oncogene 35: 4471-4480, 2016.

45. Zhang J, Wang G, Chu SJ, Zhu JS, Zhang R, Lu WW, Xia LQ, Lu YM, Da W and Sun Q: Loss of large tumor suppressor 1 promotes growth and metastasis of gastric cancer cells through upregulation of the YAP signaling. Oncotarget 7: 16180-16193, 2016.
46. Zhuang K, Yan Y, Zhang X, Zhang J, Zhang L and Han K: Gastrin promotes the metastasis of gastric carcinoma through the $\beta$-catenin/TCF-4 pathway. Oncol Rep 36: 1369-1376, 2016.

47. Guo F, Ren X, Dong Y, Hu X, Xu D, Zhou H, Meng F, Tian W and Zhao Y: Constitutive expression of PPAR $\gamma$ inhibits proliferation and migration of gastric cancer cells and down-regulates Wnt/ $\beta$-catenin signaling pathway downstream target genes TERT and ENAH. Gene 584: 31-37, 2016.

48. Fan D, Ren B, Yang X, Liu J and Zhang Z: Upregulation of miR-501-5p activates the wnt/ $\beta$-catenin signaling pathway and enhances stem cell-like phenotype in gastric cancer. J Exp Clin Cancer Res 35: 177, 2016.

49. Marti P, Stein C, Blumer T, Abraham Y, Dill MT, Pikiolek M, Orsini V, Jurisic G, Megel P, Makowska Z, et al: YAP promotes proliferation, chemoresistance, and angiogenesis in human cholangiocarcinoma through TEAD transcription factors. Hepatology 62: 1497-1510, 2015.

50. Peng Y,Zhang X, Ma Q, Yan R, Qin Y, Zhao Y, Cheng Y, Yang M, Wang Q, Feng X, et al: MiRNA-194 activates the Wnt/ $\beta$-catenin signaling pathway in gastric cancer by targeting the negative Wnt regulator, SUFU. Cancer Lett 385: 117-127, 2017.

51. Zhang B, Shi Y, Gong A, Pan Z, Shi H, Yang H, Fu H, Yan Y, Zhang $\mathrm{X}$, Wang $\mathrm{M}$, et al: HucMSC exosome-delivered 14-3-3乞 orchestrates self-control of the Wnt response via modulation of YAP during cutaneous regeneration. Stem Cells 34: 2485-2500, 2016. 